\title{
Hepatic stellate cells' involvement in progenitor-mediated liver regeneration
}

\author{
Dana G Pintilie, Thomas D Shupe, Seh-hoon Oh, Susan V Salganik, Houda Darwiche and Bryon E Petersen
}

Earlier studies conducted by our laboratory have shown that suppression of transforming growth factor- $\beta$ (TGF $\beta$ )mediated upregulation of connective tissue growth factor (CTGF) by iloprost resulted in a greatly diminished oval cell response to 2-acetylaminofluorene/partial hepatectomy $(2 \mathrm{AAF} / \mathrm{PH})$ in rats. We hypothesized that this effect is due to decreased activation of hepatic stellate cells. To test this hypothesis, we maintained rats on a diet supplemented with $2 \%$ $\mathrm{L}$-cysteine as a means of inhibiting stellate cell activation during the oval cell response to $2 \mathrm{AAF} / \mathrm{PH}$. In vitro experiments show that L-cysteine did, indeed, prevent the activation of stellate cells while exerting no direct effect on oval cells. Desmin immunostaining of liver sections from 2AAF/PH animals indicated that maintenance on the L-cysteine diet resulted in an 11.1-fold decrease in the number of activated stellate cells within the periportal zones. The total number of cells proliferating in the periportal zones of livers from animals treated with L-cysteine was drastically reduced. Further analyses showed a greater than fourfold decrease in the magnitude of the oval cell response in animals maintained on the L-cysteine diet as determined by immunostaining for both OV6 and $\alpha$-fetoprotein (AFP). Global liver expression of AFP as measured by real-time PCR was shown to be decreased 4.7-fold in the L-cysteine-treated animals. These data indicate that the activation of hepatic stellate cells is required for an appropriate oval cell response to 2AAF/PH. Laboratory Investigation (2010) 90, 1199-1208; doi:10.1038/labinvest.2010.88; published online 3 May 2010

KEYWORDS: hepatic stellate cell; L-cysteine; liver regeneration; oval cell activation; hepatic progenitor cell

Progenitor cell therapies for liver pathologies that result in massive loss of hepatic parenchyma are one alternative to liver transplantation. The 'oval cell' is the facultative hepatic progenitor cell, which aids in liver regeneration when the proliferation capacity of mature hepatocytes is compromised. These cells are bipotential (ie, capable of differentiation down both the hepatocyte and biliary lineages) and are thought to reside within the portal zones of the liver at the canals of Hering. ${ }^{1}$ A number of human conditions are associated with oval cell proliferation; among these are infections by hepatotropic viruses and terminal stages of liver cirrhosis. ${ }^{2}$ Earlier data from our laboratory suggest a coordinated interaction among the various hepatic cell types during the regeneration process, particularly between hepatic stellate cells and oval cells. ${ }^{3}$ Their co-activation has been shown in the 2-acetylaminofluorene/partial hepatectomy (2AAF/PH) model as well as several other liver remodeling processes, including hepatic fibrosis ${ }^{4}$ and liver regeneration after $\mathrm{PH}$ or D-galactosamine exposure. ${ }^{5}$ Under physiological conditions, stellate cells are quiescent, exhibiting vitamin A droplets and a star-like morphology. Their activation is followed by proliferation and differentiation into contractile myofibroblast-like cells. Known for their contribution to extracellular matrix (ECM) synthesis and remodeling, and as an important source of cytokines and growth factors, activated stellate cells are also responsible for the excessive fibrosis observed in liver cirrhosis. ${ }^{6}$ Disruption of the transforming growth factor- $\beta$ (TGF $\beta$ ) - CTGF signaling axis by the prostacyclin analogue iloprost resulted in a significant reduction of oval cell proliferation. ${ }^{3}$ As TGF $\beta$ has such a complex range of actions on various cell types, we further explored whether these effects are the result of stellate cell inhibition, or are attributable to other interactions within the regenerating liver. L-cysteine is a non-essential amino acid, widely used as a nutritional supplement, antioxidant and mucolytic agent. Recent data suggest that it might also be a potent inhibitor of liver fibrosis, acting to prevent stellate cell activation. ${ }^{7}$ Its complex effects on the liver are attributed to various

Department of Pathology, Immunology and Laboratory Medicine, and the Program for Stem Cell Biology and Regenerative Medicine, University of Florida College of Medicine, Gainesville, FL, USA

Correspondence: Associate Professor BE Petersen, PhD, Department of Pathology, Immunology and Laboratory Medicine, University of Florida College of Medicine, Box 100275, 1600, SW Archer Road, Gainesville, FL 32610-0275, USA.

E-mail: petersen@pathology.ufl.edu

Received 22 September 2009; revised 1 February 2010; accepted 1 March 2010 
mechanisms, including: synthesis of glutathione ${ }^{8,9}$; reactive oxygen species-mediated degradation of cyclin D1 with subsequent activation of manganese superoxide dismutase ${ }^{10}$; downregulation of platelet-derived growth factor receptor- $\beta$ (PDGFR $\beta)$ and inhibition of platelet-derived growth factor (PDGF) signaling. ${ }^{11,12}$ Despite the various putative mechanisms of action, it is widely accepted as a potent, harmless inhibitor of hepatic stellate cells and was proposed as an adjuvant therapy for human cirrhosis. To test our hypothesis that the stellate cells have a necessary function in facilitating oval cell proliferation, a diet supplemented with L-cysteine was combined with the well-characterized $2 \mathrm{AAF} / \mathrm{PH}$ proto$\mathrm{col}^{13,14}$ for oval cell activation in rats. This study clearly shows that hepatic stellate cell activation is required for a robust oval cell response to $2 \mathrm{AAF} / \mathrm{PH}$.

\section{MATERIALS AND METHODS}

\section{Animal Treatments}

The animal procedures involved in this study were conducted with the approval of the University of Florida IACUC. Sixweek-old Fisher 344 male rats (Charles River Laboratories, Wilmington, MA, USA) were maintained on standard laboratory chow supplemented with $2 \%$ L-cysteine (Dyets, Betlehem, PA, USA) for the duration of the experiment, according to a protocol established by Horie et al. ${ }^{7}$ One month after the initiation of the diet, $70 \mathrm{mg} 2 \mathrm{AAF}$ pellets (Innovative Research of America, Sarasota, FL, USA) were implanted intraperitoneally and, 1 week later, a $70 \% \mathrm{PH}$ was performed as described by Higgins and Anderson. ${ }^{15}$ The animals were sacrificed at defined time points as indicated in Figure 1. Tissue samples were collected and preserved by formaldehyde fixation or frozen in OCT compound (Sakura Finetek USA, Torrance, CA, USA).

\section{Cell Culture and Bromodeoxyuridine Labeling}

WB-F344 oval cells (kindly provided by Dr William Coleman) were cultured at $37^{\circ} \mathrm{C}, 5 \% \mathrm{CO}_{2}$ in RPMI- 1640 medium (Mediatech, Herndon, VA, USA) supplemented with 10\% FBS (Sigma Aldrich, St Louis, MO, USA) and 1\% penicillin-streptomycin (Mediatech). Primary isolated portal

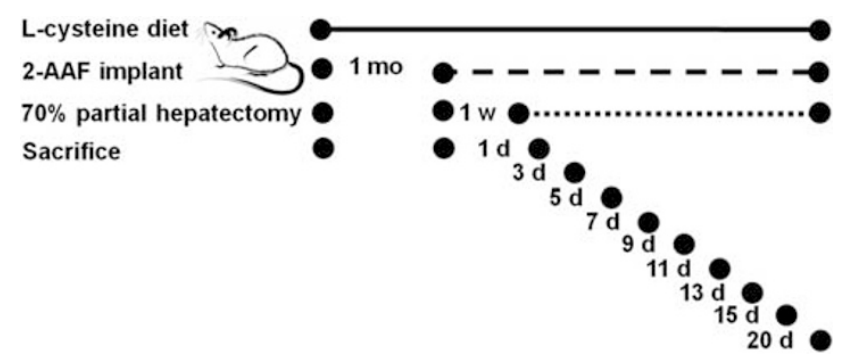

Figure 1 Study design: diagrammatic representation of the hepatic stellate cell inhibition and oval cell induction model in ô Fisher 344 rats: $2 \%$ L-cysteine diet, 2AAF pellet implantation, partial hepatectomy and selected time points for sacrifice (mo, month; w, week; d, day). fibroblasts and HSC-T6 hepatic stellate cells (kind gifts from Drs Rebecca Wells and Scott Friedman, respectively) were cultured under the same conditions in DMEM (Hyclone Lab, Logan, UT, USA) with $10 \%$ FBS and $1 \%$ penicillinstreptomycin. When $30 \%$ confluence was achieved, they were synchronized for $24 \mathrm{~h}$ with $0.4 \mu \mathrm{g} / \mathrm{ml}$ Demecolcine (Sigma Aldrich) and treated with $100 \mu \mathrm{M}$ L-cysteine (MP Biomedicals LLC, Cleveland, OH, USA), thus inhibiting the hepatic stellate cell activation. After a 3-day exposure to L-cysteine, the chamberslides were treated with $10 \mu \mathrm{M}$ bromodeoxyuridine (BrdU) (Sigma Aldrich) and fixed in $4 \%$ paraformaldehyde.

\section{Histology and Immunohistochemistry}

Paraffin embedded or frozen liver sections cut to $5 \mu \mathrm{m}$ thickness were stained with hematoxylin and eosin or immunostained using anti-OV6 (courtesy of Dr Stewart Sell), anti-Ki67 (BD Biosciences Pharmingen, Franklin Lakes, NJ, USA) or anti- $\alpha$-fetoprotein (Dako Cytochromatin, Carpinteria, CA, USA) antibodies. Anti-desmin antibody (Dako Cytochromatin) was also used for stellate cell immunostaining. Paraformaldehyde-fixed chamberslides were immunostained using anti-BrdU antibody (Dako Cytochromatin). Computer image analyses of immunostained sections were performed using Aperio ScanScope Image Analysis Platform (Aperio, Vista, CA, USA) and MetaMorph software (MDS Technologies, Concord ON, Canada) for histological evaluation and quantitation.

\section{Real-Time Quantitative PCR}

The mRNA levels were assessed by two-step quantitative real-time PCR reaction, using a DNA Engine Opticon 2 Continuously Fluorescence Detector (MJ Research, Waltham, MA, USA). Total RNA was extracted using the RNA Bee isolation kit (Tell-Test, Friendswood, TX, USA), treated with DNase I (Ambion, Austin, TX, USA) and reverse transcribed with the Superscript III First Strand Synthesis System for RT-PCR (Invitrogen, Carlsbad, CA, USA). Amplification was performed on a customized RT2Profiler PCR Array plate for the genes of interest (SA Biosciences, Frederick, MD, USA) using iQ SYBR Green Supermix (Bio-Rad Laboratories, Hercules, CA, USA). The primer pair used was: forward CAGGAGGAAGAAAGGACAAAAAA and reverse ATTCCTA AGGCATAGAAATCCCA. The amplification conditions were $10 \mathrm{~min}$ at $95^{\circ} \mathrm{C}$, followed by 40 cycles of $15 \mathrm{~s}$ at $95^{\circ} \mathrm{C}, 30 \mathrm{~s}$ at $55^{\circ} \mathrm{C}, 30 \mathrm{~s}$ at $72^{\circ} \mathrm{C}$. The comparative $\mathrm{Ct}$ threshold cycle method was used to assess the expression level, normalized to $\beta$-actin mRNA expression.

\section{Statistical Analysis}

Values were expressed as mean \pm s.d. Statistical significance was determined by analysis of variance, and Student $t$-test performed in Microsoft Excel. $P$-values $<0.05$ were considered to be statistically significant. 


\section{RESULTS}

L-Cysteine Appears to be a Selective In Vitro Inhibitor of Hepatic Mesenchymal Populations

We first examined the in vitro effects of L-cysteine on several hepatic cell populations in culture. S-phase cells were identified by BrdU incorporation into newly synthesized DNA. To exclude the possibility that L-cysteine acts directly on oval cells, the hepatic progenitor cell line, WB-F344 was cultured both with and without (Figures $2 \mathrm{a}$ and $\mathrm{d}$, respectively) $100 \mu \mathrm{M}$ L-cysteine. As expected, treatment with L-cysteine had no effect on the proliferation rate of these cells (Figure 3). We next examined primary portal fibroblast cultures (Figures 2b and e), as well as the hepatic stellate cell line HSC-T6 (Figures 2c and f). In contrast to the progenitor cell line, both of the mesenchymal cell cultures showed a significant reduction in proliferation rates when culture media was supplemented with $100 \mu \mathrm{M}$ L-cysteine. A 3.56-fold decrease in BrdU incorporation for HSC-T6 and a 5.6-fold reduction for portal fibroblasts were observed (Figures $2 \mathrm{e}$ and $\mathrm{f}$ ). Taken together, quantitative image analysis data indicate that L-cysteine acts selectively on the mesenchymal cell populations examined (Figure 3 ).

\section{Histological Changes in Oval Cell Activation Under L-Cysteine Diet}

Histological characterization of liver regeneration in the 2AAF/PH model for oval cell activation in rats showed the expected robust proliferation of small cells emanating from the periportal zone (Figures $4 \mathrm{~b}$ and e). These small oval-shaped cells were not present in untreated rat liver (Figures $4 \mathrm{a}$ and $\mathrm{e}$ ). In animals that were maintained on the $2 \% \mathrm{~L}$-cysteine diet, the small cell response in the portal zone remained quite modest (Figures $4 \mathrm{c}$ and $\mathrm{f}$ ). The disparity between the amplitude of the small cell response in the two groups was most evident on day 9 post-PH. This time point is known to coincide with the peak of oval cell proliferation after $2 \mathrm{AAF} / \mathrm{PH}$ activation protocol in rats. Aside from the reduced small cell presence in L-cysteine-treated animals, there is also a notable difference in cell morphology. In the L-cysteine-treated group, some cells tended to be larger (over $10 \mu \mathrm{m}$ diameter), with a slightly reduced nucleus to cytoplasm ratio, more rounded nuclei and basophilic vacuolar cytoplasm, bearing a resemblance to a small hepatocyte-like cell.

\section{Hepatic Stellate Cell Activation is Inhibited by L-Cysteine Diet in the 2AAF/PH Model}

Definitive identification of activated hepatic stellate cells in microscopic liver sections was accomplished by immunostaining for desmin, a cytoskeleton intermediary filament which, in rat liver, is expressed only by activated stellate cells. $^{16}$

Hepatic stellate cells are present in very low numbers in normal liver tissue (Figures $5 \mathrm{a}$ and $\mathrm{d}$ ). However, they proliferate and migrate in close proximity to the regenerating oval cells in the $2 \mathrm{AAF} / \mathrm{PH}$ model (Figures $5 \mathrm{~b}$ and e). In animals treated with L-cysteine, considerably fewer desminpositive cells are apparent after $2 \mathrm{AAF} / \mathrm{PH}$ (Figures $5 \mathrm{c}$ and $\mathrm{f}$ ).
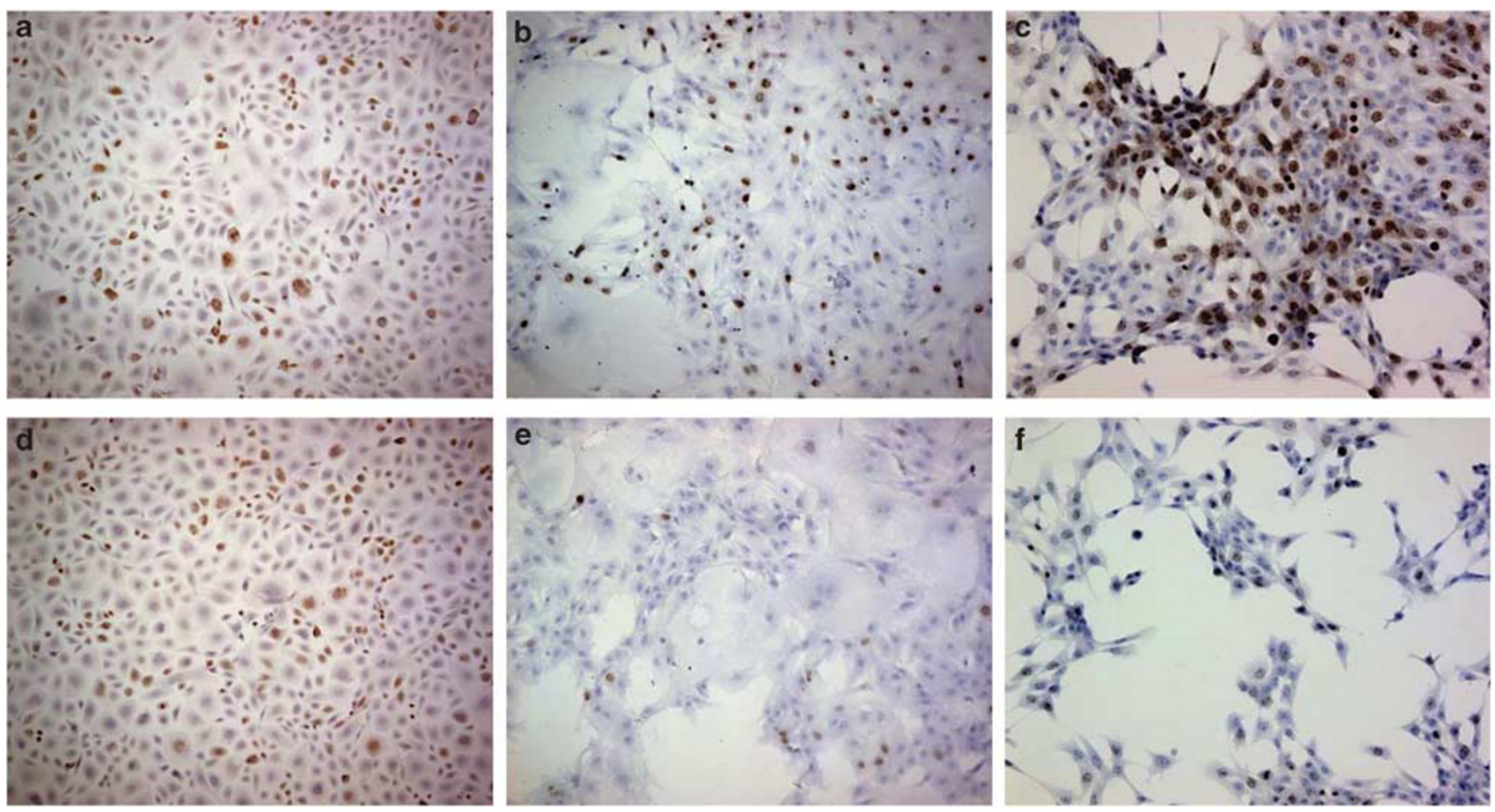

Figure 2 In vitro effects of L-cysteine on proliferation of selected hepatic cell populations: (a, d) WB-F344 cells, (b, e) primary portal fibroblasts, (c, f) HSC-T6 cells. Panels (a-c) received no treatment, whereas panels (d-f) were cultured in media supplemented with $2 \%$ L-cysteine; all shown at $\times 10$ magnification. 
Quantitative computer image analysis showed a $>11$-fold decrease in the number of desmin-positive cells in $2 \mathrm{AAF} / \mathrm{PH}$ rats maintained on the L-cysteine diet as compared with $2 \mathrm{AAF} / \mathrm{PH}$ animals that received the standard diet (Figure $5 \mathrm{~g}$ ). These results suggest that the inhibitory effects of L-cysteine on hepatic stellate cell activation work as expected in our $2 \mathrm{AAF} / \mathrm{PH}$ model of oval cell induction.

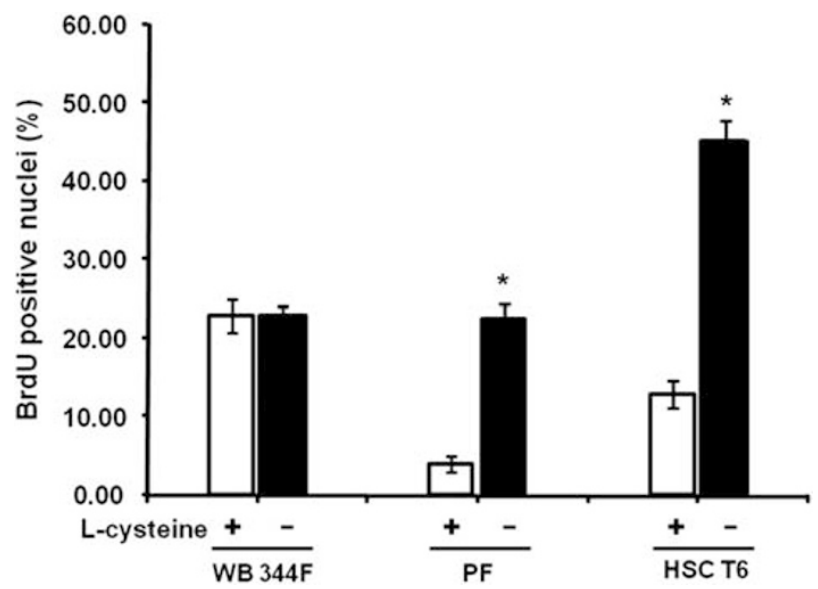

Figure $3 \mathrm{BrdU}$ index: quantitative analysis of L-cysteine effects on cultured mesenchymal and oval cells' proliferation: WB-344F, rat oval cell line; PF, primary isolated portal fibroblasts; HSC-T6, hepatic stellate cell line; white columns, cells treated with $100 \mu \mathrm{M}$ L-cysteine in culture medium; black columns, cells grown on culture medium w/out L-cysteine supplement. ${ }^{\star} P<0.05$.

\section{L-Cysteine Exposure is Associated with Reduced Cell Proliferation in Periportal Areas}

We next sought to determine the proliferation status of the cells within the periportal zones of livers from $2 \mathrm{AAF} / \mathrm{PH}$ treated animals both with, and without the L-cysteine diet. Proliferation was determined by immunostaining for Ki67 nuclear antigen on day 9 after PH. Ki67 identifies all cells that are not in $G_{0}$. In the normal liver, very few hepatocytes proliferate in the absence of an injury (Figure 6a). A large number of proliferating cells were present in the portal zones of the livers of rats subjected to the $2 \mathrm{AAF} / \mathrm{PH}$ protocol (Figure 6b). A greater than threefold reduction in the number of proliferating cells was measured in the portal zones of the livers from rats maintained on the L-cysteine diet (Figures $6 \mathrm{c}$ and d).

\section{Reduced Cell Proliferation in Periportal Areas During L-Cysteine Exposure is Associated with Diminished Desmin Presence in the Regenerating Liver}

Double immunofluorescent staining for Ki67 nuclear antigen and type III intermediate filament desmin was performed. Specifically expressed by activated stellate cells, desmin is present in portal myofibroblasts and Ito cells in normal liver (Figures $7 \mathrm{a}$ and $\mathrm{d}$ ). The numerous proliferating cells in the periportal areas of $2 \mathrm{AAF} / \mathrm{PH}$ exposed animals were surrounded by desmin-positive cytoplasmic projections (Figures $7 \mathrm{~b}$ and e). Sections collected from animals kept on L-cysteine diet exhibited a reduced presence of proliferating
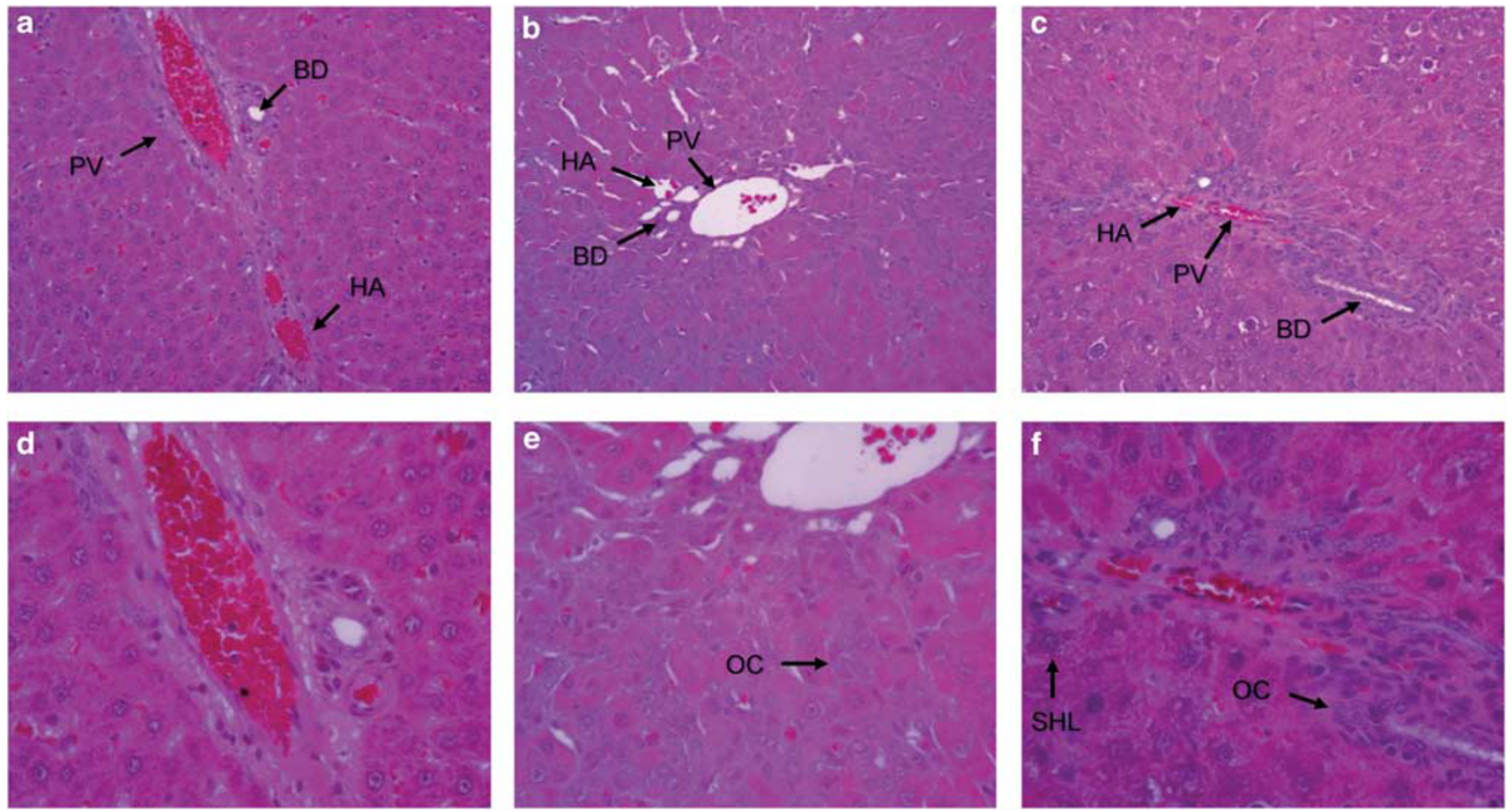

Figure 4 Comparative histological examination of hematoxylin and eosin-stained liver samples shows differences in the hepatic regeneration profile of L-cysteine-fed animals: (a, d) normal animals, (b, e) 2AAF/PH-treated rats 9 days post-PH, (c, f) L-cysteine/2AAF/PH protocol 9 days after acute liver injury. BD, bile duct; PV, portal vein branch; HA, hepatic artery branch; OC, oval cell; SHL, small hepatocyte-like cell. Upper panels are shown at $\times 20$, lower panels at $\times 40$ magnification. 

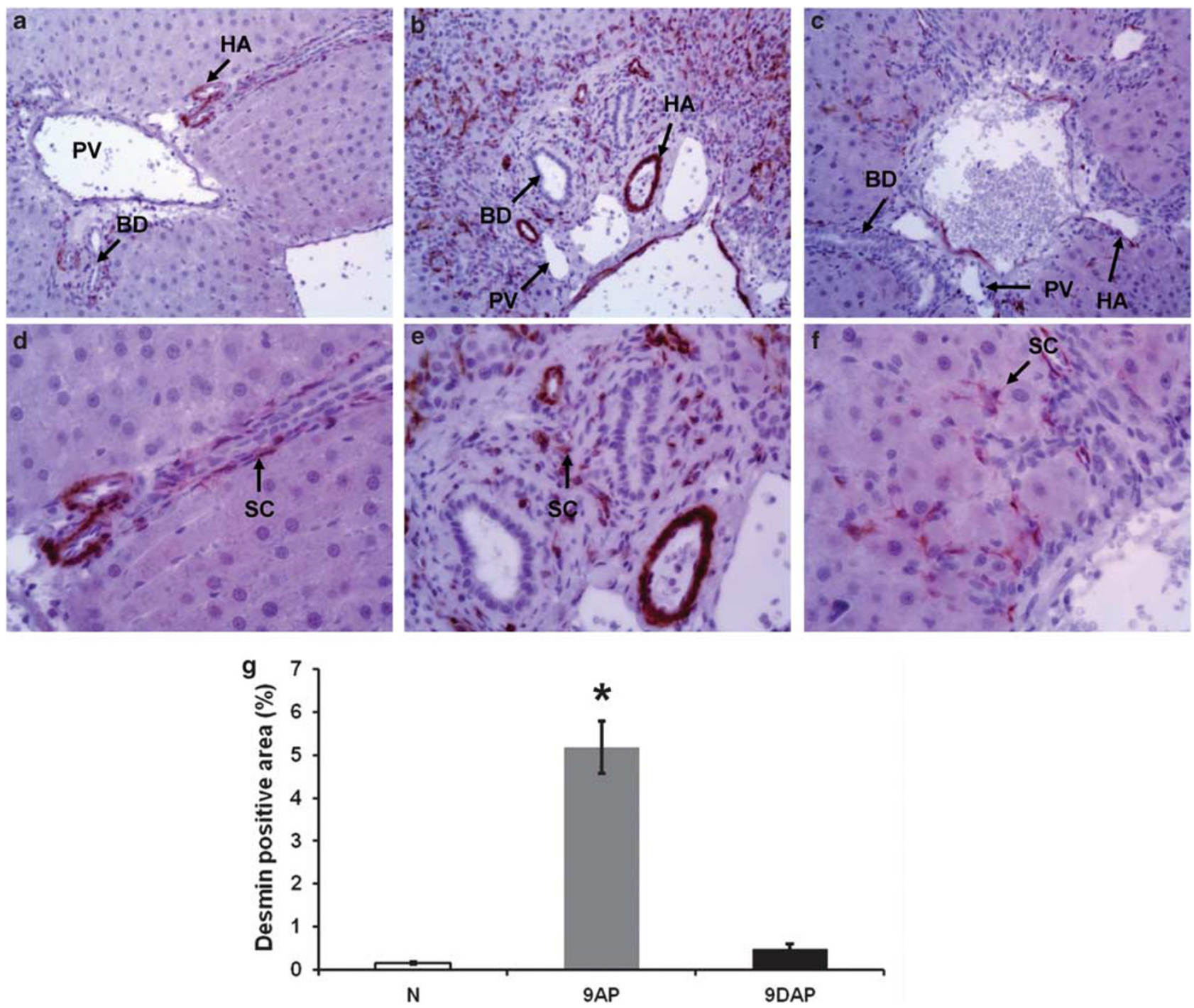

Figure 5 In vivo reduced activation of hepatic stellate cell population indicated by desmin immunostaining of liver sections from L-cysteine-fed rats: normal rat liver (a, d), N; liver at day 9 after 2AAF/PH (b, e), 9AP; same time point liver section from animals subjected to L-cysteine diet/2AAF/PH (c, f), 9DAP. $\mathrm{BD}$, bile duct; PV, portal vein branch; HA, hepatic artery branch; SC, stellate cell. Upper panels are shown at $\times 20$, lower panels at $\times 40$ magnification. (g) Quantitative computer image analysis of desmin-positive areas on the immunostained liver samples confirms the significant reduction of stellate cell activation on immunostained sections. ${ }^{*} P<0.05$.

cells accompanied by few desmin-positive cells in acinar zone I of regenerating liver (Figures $7 \mathrm{c}$ and $\mathrm{e}$ ).

\section{Regenerating Cells in the Periportal Areas are AFP and ov6 Positive}

To identify the oval cell population within the portal zone on day 9 after $2 \mathrm{AAF} / \mathrm{PH}$, paraffin sections were stained for $\alpha$-fetoprotein (AFP). On the normal liver sections, no AFPpositive cells were apparent (Figures $8 \mathrm{a}$ and $\mathrm{d}$ ). As expected, the portal zones of livers from $2 \mathrm{AAF} / \mathrm{PH}$-treated animals contained a large population of AFP-positive cells (Figures $8 \mathrm{~b}$ and e). Animals exposed to L-cysteine showed a greater than fourfold decrease in AFP-positive cells (Figures 8c, $\mathrm{f}$ and $\mathrm{g}$ ).
It is worth noting that a greater percentage of the cells within the portal zones of livers from animals maintained on the L-cysteine diet appeared to be transitional hepatocytes (ie, larger, hepatocyte-like cells with weak expression of AFP).

Global AFP expression in the livers of animals subjected to $2 \mathrm{AAF} / \mathrm{PH}$ was determined by quantitative real-time PCR (Figure 9a). AFP message was measured relative to the normal liver and normalized to $\beta$-actin. Animals that were exposed to L-cysteine showed a 4.7 -fold decrease in total liver expression of AFP as compared with animals that were fed the normal diet. These results suggest a significant reduction of oval cell contribution to hepatic mass recovery. 

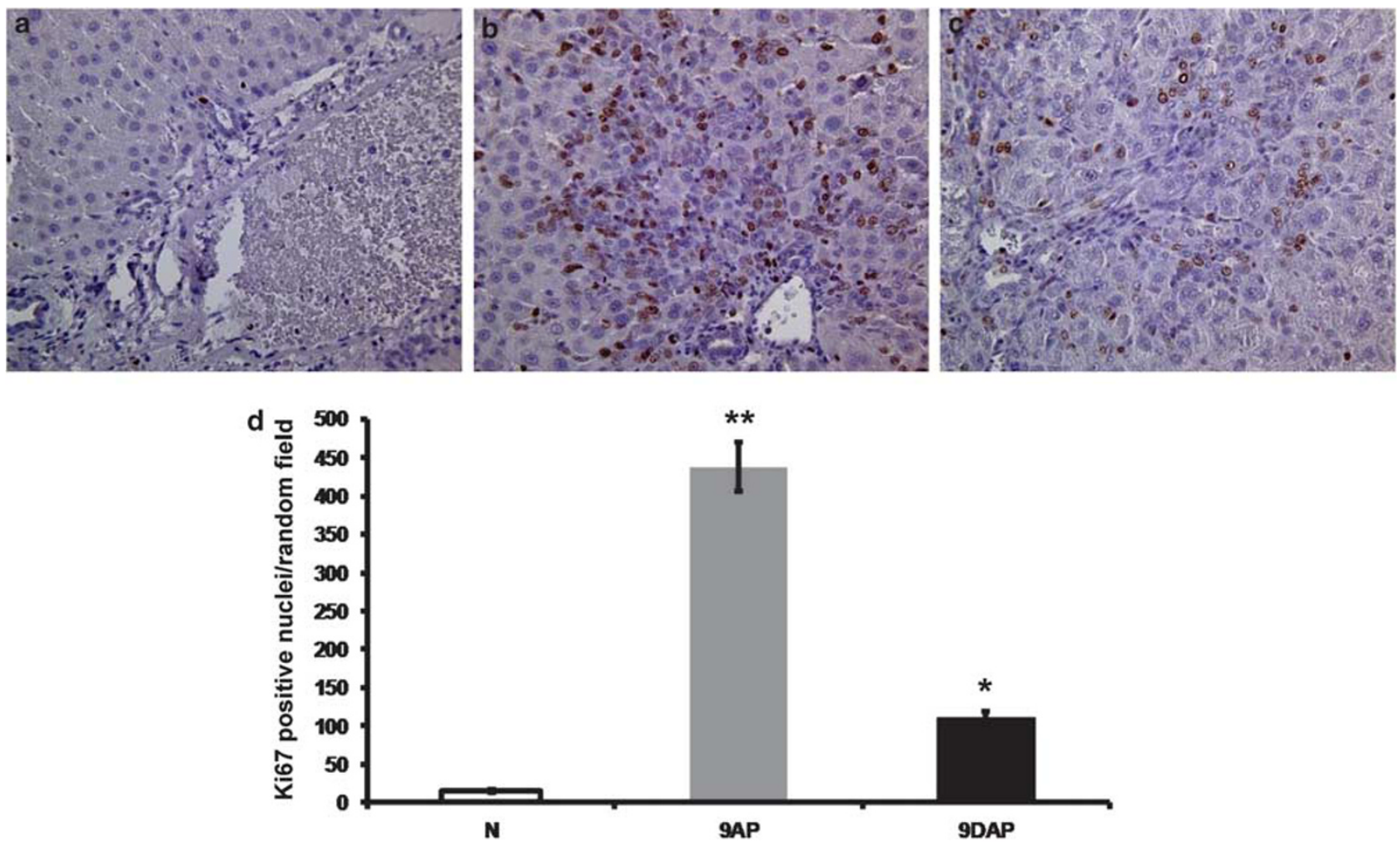

Figure 6 Assessment on proliferation status of hepatic cell populations by quantitative analysis of Ki67-positive nuclei on random microscopic fields: normal liver (a), N, samples collected 9 days after the acute liver injury (b), 9AP, rats subjected to the 2AAF/PH protocol (c), 9DAP, and fed with L-cysteine during the oval cell activation treatment. All shown at $\times 20$ magnification. Quantitative computer image analysis of Ki67 positive nuclei confirms the significant reduction of hepatic cell proliferation (d). ${ }^{\star} P<0.05 ;{ }^{* *} P<0.005$
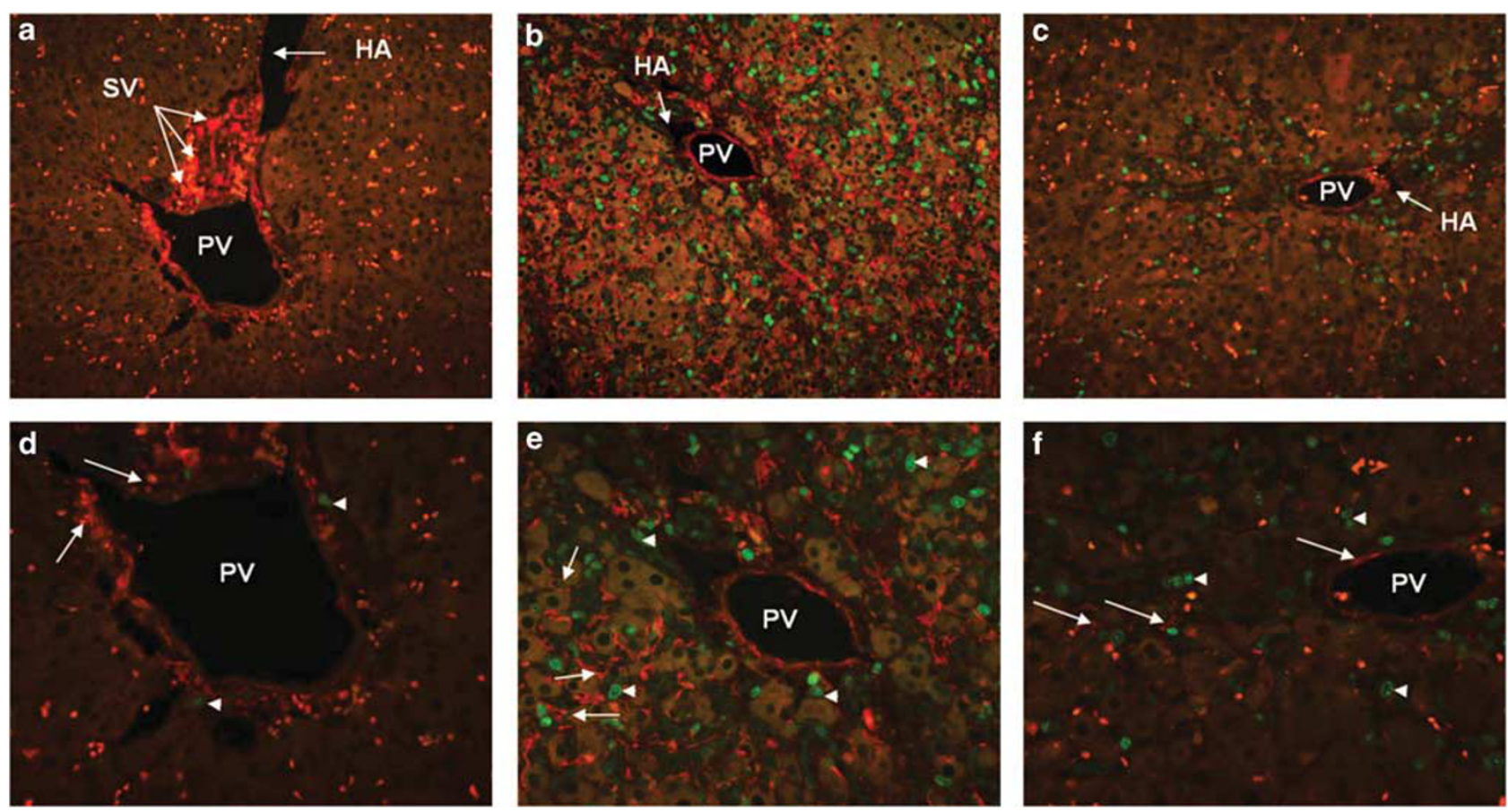

Figure 7 Concomitant reductions in proliferation of hepatic cell populations and oval cell activation, reflected by Ki67 and desmin-immunofluorescent staining, respectively: normal liver $(\mathbf{a}, \mathbf{d})$; sections collected 9 days post-hepatectomy from animals subjected to $2 \mathrm{AAF} / \mathrm{PH}$ protocol (b, e) and rats maintained on L-cysteine/2AAF/PH (c, f). PV, portal vein branch; HA, hepatic artery branch. Upper panels are shown at $\times 20$, lower panels are shown at $\times 40$ magnification. 

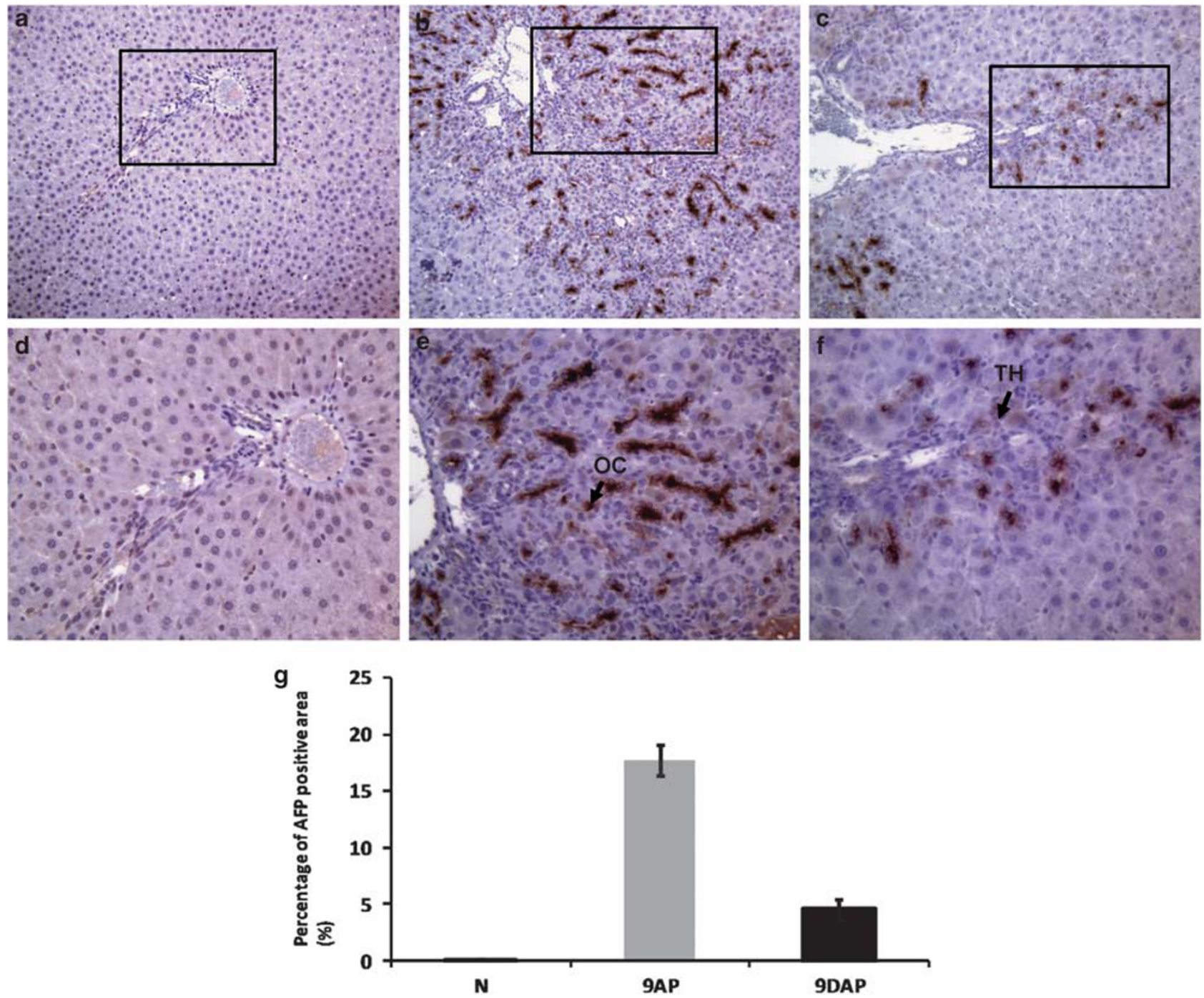

Figure 8 Reduced oval cell presence on AFP immunostained liver sections suggested by quantitative image analysis (g): normal liver (a, d), N, 2AAF/PHtreated rats day 9 after $\mathrm{PH}(\mathbf{b}, \mathbf{e}), 9 \mathrm{AP}$, and 2AAF/PH-treated rats maintained on the $2 \%$ L-cysteine diet 9 days after PH (c, f), 9DAP. OC, oval cell; TC, transitional hepatocytes. Upper panels are shown at $\times 10$, lower panels are shown at $\times 20$ magnification.

An alternative oval cell marker was used to confirm the findings of the AFP immunostaining. OV6 is a wellcharacterized marker for oval cells and bile duct cells. In normal liver, only the bile ducts within the portal triad were positive for OV6 (Figures 10a and d). Liver samples from animals subjected to $2 \mathrm{AAF} / \mathrm{PH}$ contained a large population of OV6-positive cells within the periportal zone that radiated out toward the central vein (Figures $10 \mathrm{~b}$ and e). This is consistent with a normal oval cell response at day 9 after $\mathrm{PH}$ in the $2 \mathrm{AAF} / \mathrm{PH}$ model. In contrast to this, the liver sections from animals maintained on the L-cysteine diet displayed a very modest oval cell response at day 9 after $\mathrm{PH}$ (Figures 10c and $\mathrm{f}$ ). Computer image analysis of scanned slides confirmed the 3.5-fold disparity in the magnitude of oval cell response in animals that were fed the normal rat food, as compared with animals that were administered L-cysteine (Figure 10g).

\section{DISCUSSION}

Progenitor cell-mediated liver regeneration is an alternative compensatory hyperplasia, able to restore hepatic mass when hepatocyte proliferation is severely impaired by massive liver necrosis or chronic cirrhogenic conditions. ${ }^{17}$ Oval cells and stellate cells are among the first cells to enter the cell cycle after $2 \mathrm{AAF} / \mathrm{PH}$ progenitor activation protocol. $^{18}$ Less is known regarding the exact temporal relationship between oval and stellate cell activation in the regenerating liver. It is for this reason that we chose to begin the L-cysteine diet well before the initiation of the $2 \mathrm{AAF} / \mathrm{PH}$ protocol (Figure 1). The daily food intake and the body weight of all animals were monitored for the duration of the study to identify any potential effects attributable to the diet, and no significant differences were seen between animals fed the experimental and control diets. Our in vitro studies also show that 


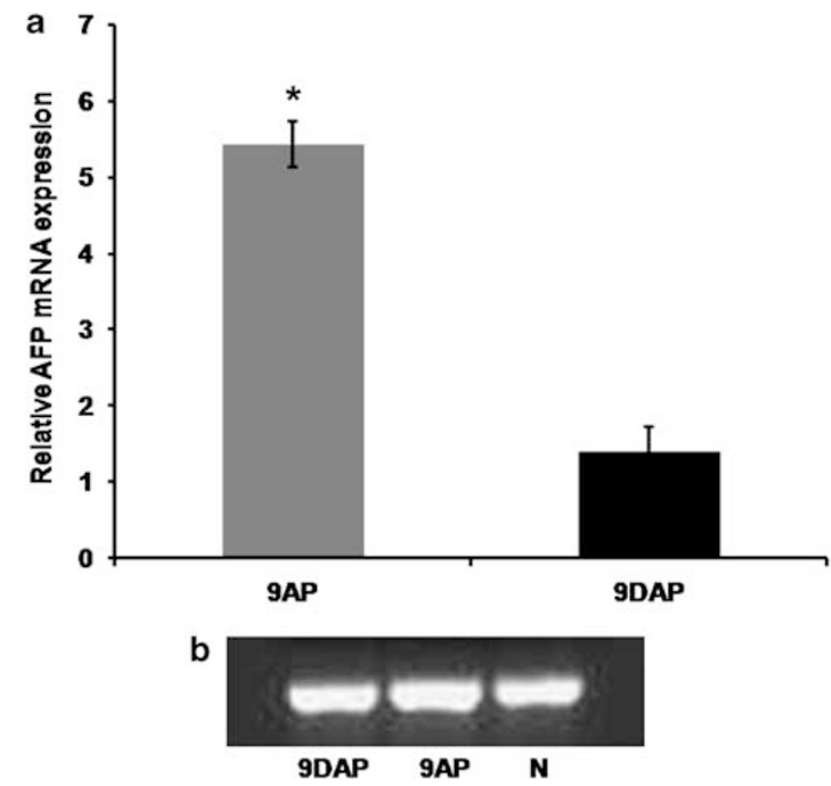

Figure 9 Magnitude of oval cell response quantified by real-time PCR analysis of AFP mRNA, relative to normal liver tissue expression and normalized to $\beta$-actin (a), 9AP, animals fed normal rat chow and subjected to 2AAF/PH protocol; 9DAP, animals maintained on the L-cysteine diet associated to $2 \mathrm{AAF} / \mathrm{PH}$ treatment. GAPDH was used as a loading control (b). ${ }^{\star} P<0.05$.

L-cysteine does not directly govern oval cell proliferation; nor does it induce any alterations of their phenotype (Figure 2). Taken together, these facts indicate that the effects of L-cysteine in our model result from the specific inhibition of hepatic stellate cells.

The hepatocyte proliferation inhibitor 2AAF is activated and detoxified by the liver through rounds of hydroxylation and conjugation, ${ }^{19}$ which lead to renal excretion of watersoluble derivatives. ${ }^{20,21}$ Being a precursor for glutathione synthesis, it is reasonable to consider the possibility that L-cysteine could increase the rate of 2AAF detoxification. This would potentially lead to incomplete suppression of hepatocyte proliferation in the $2 \mathrm{AAF} / \mathrm{PH}$ model. However, examination of Ki67-stained liver sections from the L-cysteine-treated group showed no signs of mature hepatocyte proliferation, the only Ki67-positive cells being oval cells and small hepatocyte-like phenotype (Figure 6). This would seem to exclude differential 2AAF metabolism as a complicating factor in these studies. The reduced Ki67 presence in the animals maintained on L-cysteine was associated with a diminished presence of desmin in the periportal spaces, indicating a reduced activation of hepatic stellate cells. Under these circumstances, the number of proliferating cells in the periportal areas was also reduced, suggesting an association between a reduced contribution of stellate cells and diminished hepatic regeneration (Figures $7 \mathrm{c}$ and $\mathrm{f}$ as opposed to Figures $7 \mathrm{~b}$ and $\mathrm{e})$.

Progenitor cell-mediated liver regeneration is a complex process, involving sequential waves of cytokine secretion and remodeling of the ECM. These two processes are intimately coupled, as the matrix can liberate chemical signals when degraded, and concentrate chemical signals ${ }^{22}$ that bind to the matrix within specific regions. ${ }^{23,24}$ Thus, ECM functions as a primary reservoir of biologically active molecules in the liver. ${ }^{18}$ During hepatic regeneration, the presence of increased numbers of hepatic stellate cells was noted in close proximity to the oval cells. ${ }^{25}$ Activated hepatic stellate cells are the main source of MMPs and TIMPS that participate in matrix remodeling and release of bound cytokines. ${ }^{18,26}$ Matrix remodeling results in the establishment of a unique microenvironment, conducive for the proliferation and migration of cells within the regenerating zone. This renders the activation of hepatic stellate cells a critical component of progenitor cell-mediated liver regeneration process. Earlier research conducted by our group shows that during progenitor cell-mediated liver regeneration, a fibronectin-rich provisional matrix is synthesized in the portal zone. ${ }^{23}$ We feel that it is likely that this provisional matrix contributes to the oval cell response, acting as a required substrate on which oval cells may proliferate and providing binding sites for signaling molecules that regulate oval cell phenotype. One such example is CTGF, which binds to the fibronectin-rich provisional matrix of the periportal zone, where it is concentrated and made available to the oval cells, which are known to express CTGF receptors. ${ }^{23}$

Several recently published studies clearly show that stellate cells within the liver may, through a process of mesenchymal to epithelial transition, give rise to hepatocytes. ${ }^{27}$ It is possible that this phenomenon involves an intermediate cell type with oval cell properties. It is impossible to determine whether decreased mesenchymal to epithelial transition contributes to the reduction in oval cell proliferation seen in our model. However, this possibility deserves mention.

Another interesting feature of the L-cysteine-treated livers is the apparent accumulation of transitional or intermediate hepatocytes (Figure 4), displaying a phenotype characteristic for the regeneration process, without any alterations induced by the diet. These cells are morphologically similar to hepatocytes, but are much smaller and weakly express AFP. In our model, we speculate that these cells are oval cells that have failed to complete the differentiation program, but at this stage, the absence of reliable markers has made their precise identification impossible. This would suggest that inhibition of stellate cell activation after $2 \mathrm{AAF} / \mathrm{PH}$ not only affects oval cell proliferation, but also oval cell differentiation. Once again, this would most likely result from the lack of an appropriate microenvironment within the regenerating zone and advocates the 'nurturing role' of stellate cells during the regeneration process. Although the cellular proliferation appears to be attenuated quantitatively in response to stellate cell inhibition, we have not noticed any phenotype alterations of the oval cells during the regeneration process. Overall, the blunted oval cell response in animals fed the L-cysteine diet is likely due to a combination of the loss of a major cytokine 

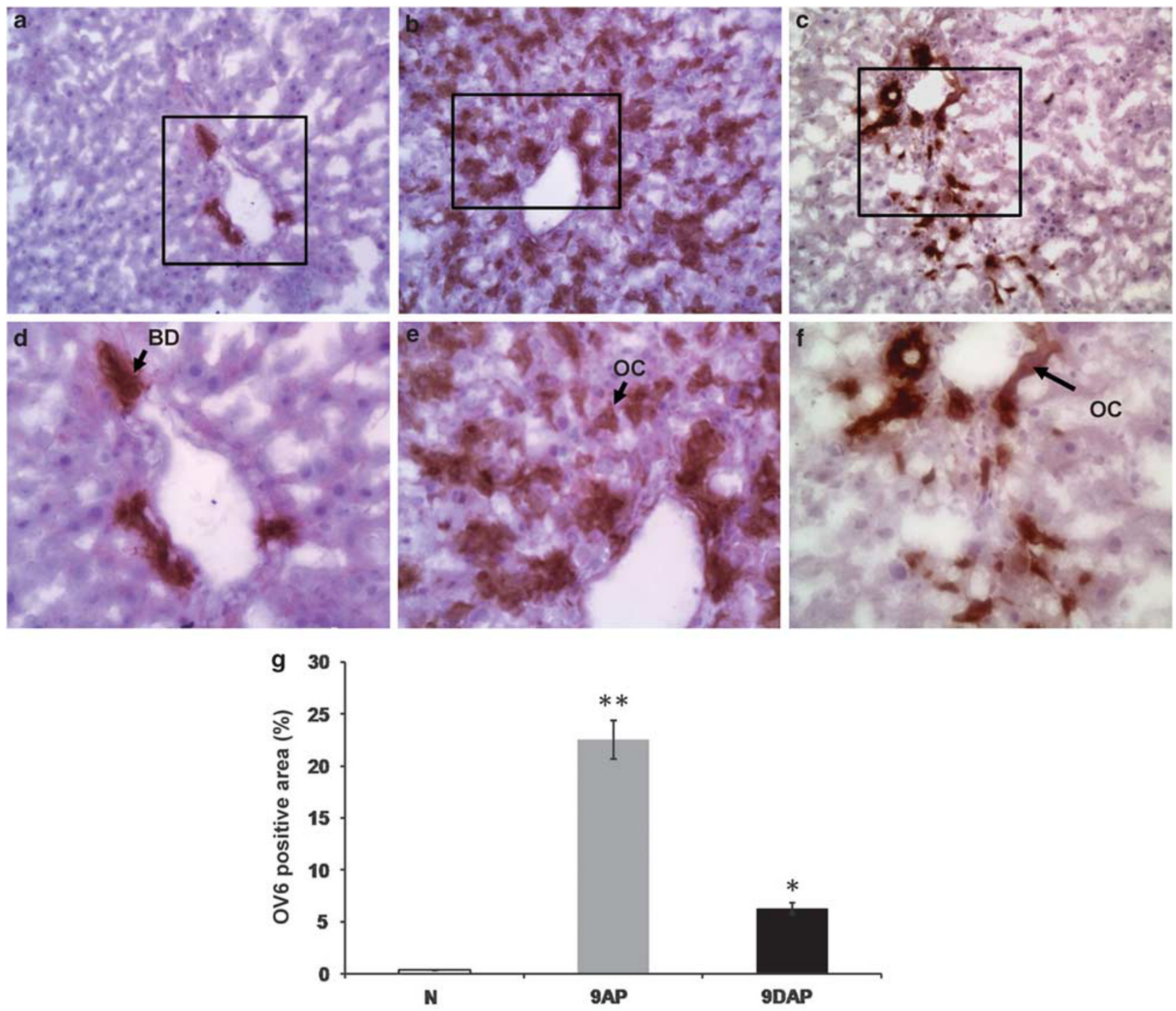

Figure 10 Oval cell response reduction assessed by quantitative image analysis ( $\mathbf{g}$ ) of OV6-positive areas on tissue sections obtained from normal liver $(\mathbf{a}, \mathbf{d}), \mathrm{N}$, and animals killed 9 days after the acute liver injury; (b, e) 9AP, subjected to the 2AAF/PHx protocol and (c, f) 9DAP, fed L-cysteine during the oval cell activation treatment. OC, oval cell; BD, bile duct. Upper panels are shown at $\times 20$, lower panels are shown at $\times 40$ magnification. ${ }^{\star} P<0.05$; ${ }^{*} P<0.005$.

source (ie, activated stellate cells), coupled with the loss of an appropriate microenvironment for the expansion of the oval cell population (ie, the fibronectin-rich provisional matrix). The end result of L-cysteine treatment is a rough doubling of the time required for the regeneration of the liver after $2 \mathrm{AAF} /$ $\mathrm{PH}$ (data not shown). As the injury does eventually resolve in the L-cysteine-treated group, redundant pathways for the regulation of oval cell phenotype likely exist. However, the significant reduction in the oval cells seen at what would normally be considered the time of maximal oval cell proliferation proves, for the first time, the critical role of stellate cells in oval cell-mediated liver regeneration.

\section{ACKNOWLEDGEMENTS}

We thank Dr Rebecca Wells from the University of Pennsylvania, Dr William Coleman from the University of North Carolina and Dr Scott Friedman from
Mount Sinai School of Medicine for their kind gifts of primary isolated portal fibroblasts, WB-F344 cells and HSC-T6 cells, respectively, and to Dr Stuart Sell from Ordway Research Institute for generously providing the OV6 antibody. Grant support DK58614, DK65096 was awarded to BEP.

\section{DISCLOSURE/CONFLICT OF INTEREST}

The authors declare no conflict of interest.

1. Kuwahara R, Kofman AV, Landis CS, et al. The hepatic stem cell niche: identification by label-retaining cell assay. Hepatology 2008;47:1994-2002.

2. Xiao JC, Jin XL, Ruck $P$, et al. Hepatic progenitor cells in human liver cirrhosis: immunohistochemical, electron microscopic and immunofluorescence confocal microscopic findings. World J Gastroenterol 2004;10:1208-1211.

3. Pi L, Oh SH, Shupe T, et al. Role of connective tissue growth factor in oval cell response during liver regeneration after $2-\mathrm{AAF} / \mathrm{PHx}$ in rats. Gastroenterology 2005;128:2077-2088. 
4. Friedman SL. Liver fibrosis-from bench to bedside. J Hepatol 2003; 38(Suppl 1):S38-S53.

5. Ujike K, Shinji T, Hirasaki S, et al. Kinetics of expression of connective tissue growth factor gene during liver regeneration after partial hepatectomy and D-galactosamine-induced liver injury in rats. Biochem Biophys Res Commun 2000;277:448-454.

6. Friedman SL. Mechanisms of hepatic fibrosis and therapeutic implications. Nat Clin Pract Gastroenterol Hepatol 2004;1:98-105.

7. Horie T, Sakaida I, Yokoya F, et al. L-cysteine administration prevents liver fibrosis by suppressing hepatic stellate cell proliferation and activation. Biochem Biophys Res Commun 2003;305:94-100.

8. Kim KI, Rhim T, Choi l, et al. N-acetylcysteine induces cell cycle arrest in hepatic stellate cells through its reducing activity. J Biol Chem 2001;276:40591-40598.

9. Zheng S, Yumei F, Chen A. De novo synthesis of glutathione is a prerequisite for curcumin to inhibit hepatic stellate cell (HSC) activation. Free Radic Biol Med 2007;43:444-453.

10. Menon SG, Sarsour EH, Kalen AL, et al. Superoxide signaling mediates $\mathrm{N}$-acetyl-L-cysteine-induced G1 arrest: regulatory role of cyclin D1 and manganese superoxide dismutase. Cancer Res 2007;67:6392-6399.

11. Okuyama $\mathrm{H}$, Shimahara $\mathrm{Y}, \mathrm{Kawada} \mathrm{N}$, et al. Regulation of cell growth by redox-mediated extracellular proteolysis of platelet derived growth factor receptor b. J Biol Chem 2001;276:28274-28280.

12. Matsui $H$, Ikeda $K$, Nakajima $Y$, et al. Sulfur-containing amino acids attenuate the development of liver fibrosis in rats through downregulation of stellate cell activation. J Hepatol 2004;40:917-925.

13. Solt DB, Farber E. New principle for the analysis of chemical carcinogenesis. Nature (Lond) 1976;263:702-703.

14. Petersen BE, Goff JP, Greenberger JS, et al. Hepatic oval cells express the hematopoietic stem cell marker Thy- 1 in the rat. Hepatology 1998;27:433-445.

15. Higgins GM, Anderson RM. Experimental pathology of the liver. Arch Pathol 1931;12:186-201.

16. Tsusumi M, Takada A, Takase S. Characterization of desmin-positive rat liver sinusoidal cells. Hepatology 1987;7:277-284

17. Lowes KN, Brennan BA, Yeoh GC, et al. Oval cell numbers in human chronic liver diseases are directly related to disease severity. Am J Pathol 1999;154:537-541.
18. Evarts RP, Nakatsukasa $\mathrm{H}$, Marsden ER, et al. Expression of transforming growth factor-a in regenerating liver and during hepatic differentiation. Mol Carcinog 1992;5:25-31.

19. Miller EC, Miller JA, Hartmann HA. N-hydroxy-2-acetylaminofluorene: a metabolite of 2-acetylaminofluorene with increased carcinogenic activity in the rat. Cancer Res 1961;21:815-824.

20. Weisburger JH, Weisburger EK. Biochemical formation and pharmacological, toxicological, and pathological properties of hydroxylamines and hydroxamic acids. Pharmacol Rev 1973;25:1-66. Review.

21. Lotlikar PD, Hong YS. Microsomal N- and C-oxidations of carcinogenic aromatic amines and amides. Natl Cancer Inst Monogr 1981; 58:101-107.

22. Ellerbroek SM, Wu YI, Stack MS. Type I collagen stabilization of matrix 695 metalloproteinase-2. Arch Biochem Biophys 2001;390: 51-56.

23. Pi L, Ding $X$, Jorgensen $M$, et al. Connective tissue growth factor with a novel fibronectin binding site promotes cell adhesion and migration during rat oval cell activation. Hepatology 2008;47: 996-1004.

24. Freise $C$, Erben $U$, Muche $M$, et al. The alpha 2 chain of collagen type VI sequesters latent proforms of matrix-metalloproteinases and modulates their activation and activity. Matrix Biol, print copy in press 2009 August. Available from URL: www.sciencedirect.com/science?_ob = ArticleURL\&_udi $=$ B6VPM-4X1SB84-2\&_user $=2139813 \& \_$rdoc $=1$ $\& \_\mathrm{fmt}=$ \&_orig $=$ search \&_sort $=\mathrm{d} \& \_$docanchor $=\& \mathrm{view}=\mathrm{c} \& \_\mathrm{acct}=$ C000054276\&_version $=1 \&$ \&urlVersion $=0 \&$ _userid $=2139813 \& \mathrm{md} 5=$ f0621d8c889048dbf732074d8db11c6b.

25. Paku S, Schnur J, Nagy $P$, et al. Origin and structural evolution of the early proliferating oval cells in rat liver. Am J Pathol 2001;158: 1313-1323.

26. Knittel T, Mehde M, Kobold D, et al. Expression patterns of matrix metalloproteinases and their inhibitors in parenchymal and nonparenchymal cells of rat liver: regulation by TNF-alpha and TGF-beta1. J Hepatol 1999;30:48-60.

27. Yang $L$, Jung $Y$, Omenetti $A$, et al. Fate-mapping evidence that hepatic stellate cells are epithelial progenitors in adult mouse livers. Stem Cells 2008;26:2104-2113. 